\title{
Cerebellar ataxia, areflexia, pes cavus, optic atrophy, and sensorineural hearing loss (CAPOS): a new syndrome
}

\author{
P Nicolaides, R E Appleton, A Fryer
}

\begin{abstract}
There are a large number of well recognised syndromes comprising cerebellar ataxia in association with other neurological features. We report three family members who presented with a relapsing, early onset cerebellar ataxia, associated with progressive optic atrophy and sensorineural deafness. All three patients have areflexia (in the absence of a peripheral neuropathy), a pes cavus deformity, and show varying degrees of severity. Extensive neurological investigations have been normal, and the aetiology and pathophysiology of this disorder remain unclear. This may represent a separate syndrome of early onset cerebellar ataxia with associated features ("cerebellar ataxia plus"), which is likely to either have an autosomal dominant or maternal mitochondrial pattern of inheritance. The recognition of this association under the acronym of CAPOS (cerebellar ataxia, areflexia, pes cavus, optic atrophy, and sensorineural deafness) may help in the delineation of a new syndrome.

( $f$ Med Genet 1996;33:419-421)
\end{abstract}

Key words: ataxia; optic atrophy; deafness.

Cerebellar ataxia is an infrequent isolated finding in otherwise normal healthy people and families. Its association with other neurological abnormalities is well recognised, and has resulted in the emergence of the term "ataxia plus". ${ }^{1}$ A number of these ataxic disorders result from known inherited metabolic defects while others are of undetermined aetiology. In this paper we describe a family with what appears to be a new dominantly inherited ataxic syndrome with a relapsing course, characterised by early onset cerebellar ataxia, areflexia, pes cavus, optic atrophy, and sensorineural deafness (CAPOS).

The Royal Liverpool Children's NHS Trust, Alder Hey, Liverpool L12 2AP, UK $P$ Nicolaides R E Appleton A Fryer

Correspondence to: Dr Nicolaides, The Roald Dahl EEG Unit, The Roya Liverpool Children's NHS Trust, Alder Hey, Liverpool L12 2AP, UK.

Received 15 September 1995

Revised version accepted for publication 4 January 1996

\section{Case reports}

CASE 1

This boy was the first child of non-consanguineous parents. He was born normally at term with a birth weight of $3100 \mathrm{~g}$ and achieved normal developmental milestones including independent walking at the age of 1 year.

He first presented at the age of 16 months with a one day history of a non-specific febrile illness, following which he developed generalised hypotonia and cerebellar ataxia. This episode resolved over the next few days and he made a full recovery within three weeks from presentation. At the age of 2 years he experienced a similar febrile illness which was associated with drowsiness and lethargy and this lasted for a week. Neurological examination at that time showed cerebellar ataxia, dysarthria, areflexia, flexor plantar responses, and pes cavus. His ataxia improved over the following months, although it tended to worsen slightly in association with any intercurrent illness. From 3 years of age he developed visual and auditory impairment. At the age of $4 \frac{1}{2}$ years, he had his third and most severe relapse, again in association with a mild, non-specific febrile illness, in which he remained unrousable for a week, followed by a very slow recovery. He was unable to sit up or walk for many months owing to marked hypotonia and ataxia. He required intensive physiotherapy and occupational and speech therapy. Six months after this relapse he was able to walk independently. Currently aged 6 years, his gait remains markedly ataxic, he has poor hand coordination, truncal hypotonia, nystagmus, dysarthria, and has developed progressive optic atrophy and sensorineural deafness. He has a bilateral $50 \mathrm{~dB}$ sensorineural hearing loss at $500 \mathrm{~Hz}$ and a $20 \mathrm{~dB}$ loss at $1000 \mathrm{~Hz}$, with a normal tympanogram, and has bilateral hearing aids. Visual acuities have progressively reduced over the course of one year from $6 / 9$ on the right, $6 / 12$ on the left to $6 / 60$ bilaterally and from N5 right, N6 left to N24 bilaterally. Numerous investigations have yielded normal or negative results (table). Electrophysiology showed a normal electroretinogram (ERG), but absent pattern reversal visual evoked potentials (VEPs) from both eyes with reduced amplitude flash VEPs. Following his latest relapse he has been treated with acetazolamide in an attempt to prevent a recurrence, should he have an "atypical" form of acetazolamide responsive familial periodic ataxia.
CASE 2

His mother, aged 31 years, has mild cerebellar ataxia, absent deep tendon reflexes, pes cavus, profound (50-60 dB) sensorineural deafness, horizontal nystagmus, and bilateral optic atrophy. She was well until the age of 18 months when she developed acute ataxia during a febrile illness. She showed some improvement but did not make a full recovery; her signs were ascribed to a "viral illness". She has experienced no further relapses but has shown 


\section{Normal/negative investigations in case 1}

Blood Full blood count, erythrocyte sedimentation rate, liver enzymes, thyroid function, autoantibodies, ammonia, urate, cholesterol, triglycerides, apoproteins A and B, vitamins and E, $\alpha$ fetoprotein, chromosome fragility studies, immunoglobulins and IgG subclasses, amino acids, very long chain fatty acids, bile acids, white cell enzymes, vitamin B12 and folate, phytanic acid, copper and caeruloplasmin, absent vacuolated lymphocytes and acanthocytes, DNA analysis for spinocerebeller ataxia type 1 (SCA 1) and for mitochondrial cytopathies (MELAS, MERRF, NARP, LHON) and vir (SCA 1) ar

Urine Amino acids, organic acids, porphyria screen (all repeated $\times 2$, including during relapses).

relapses)

Cerebrospinal fluid (CSF) Protein, amino acids, lactate, glucose, cell count, bacterial culture and viral antibody titres.

Cranial imaging CT (aged 2 years) and MRI (aged 4.5 years)

Biopsies Skin fibroblast culture for pyruvate dehydrogenase (PDH) $0.83 \mathrm{nmol} / \mathrm{mg}$ protein $/ \mathrm{min}$ $(0 \cdot 7-1 \cdot 2)$ and pyruvate carboxylase.

Muscle histology: normal mitochondria, no ragged red fibres seen;

respiratory chain enzyme studies (complexes I, II, III, IV) and mitochondrial DNA deletions normal.

Electrocardiogram and echocardiography

Electroencephalogram

Nerve conduction (velocities and amplitude of responses) and electromyography (aged 2 and 4.5 years)

Electroretinogram

MELAS = mitochondrial encephalomyopathy, lactic acidosis, stroke-like episodes MERRF $=$ myoclonic epilepsy, ragged red fibres.

NARP $=$ neurogenic muscle weakness, ataxia, retinitis pigmentosa.
LHON = Leber's hereditary optic neuropathy. a progressive course with visual impairment, deafness, and loss of balance. She has one other daughter described below (case 3); there have been no other pregnancies. Electrophysiological studies showed normal somatosensory evoked potentials (SSEP) and ERG, but severely abnormal pattern and borderline flash VEPs, in addition to absent brain stem/auditory evoked potentials (BSAEP) from either ear. There were normal nerve conduction velocities (NCVs), with no evidence of a peripheral neuropathy. Electromyography (EMG) showed evidence of some motor unit loss and reinnervation changes in the tibialis anterior. Cranial magnetic resonance imaging (MRI) at the age of 31 years is normal.

CASE 3

The proband's previously well 2 year old sister had a non-specific febrile illness at the age of 9 months, which was accompanied by marked hypotonia, nystagmus, ataxia, areflexia, and flexor plantar responses. She made a complete recovery over three days but has persisting horizontal nystagmus, mild, generalised hypotonia, areflexia, and early optic atrophy with a visual acuity of $6 / 9$ in the right and $6 / 12$ in the left eye. Her hearing responses to low frequency sounds were significantly raised. Cranial MRI and biochemical/metabolic investigations similar to those undertaken in her brother have been normal.

None of these three patients have any history of epileptic seizures, intellectual impairment, or other medical disorder. There are two maternal sibs (a sister aged 34 years and a brother aged 30 years) who are both unaffected, and the grandparents have never experienced any similar episodes and have a normal neurological examination.

\section{Discussion}

The classification of cerebellar ataxic syndromes is complex and based on age of onset, natural history (static, progressive, or relapsing), aetiology or the presence of associated features or both. ${ }^{1}$ Many of these "ataxic" syndromes are rare, poorly defined, and are frequently only described in the members of a single family. Autosomal dominant, autosomal recessive, $\mathrm{X}$ linked recessive, and mitochondrial inheritance patterns have all been described.

Our family exhibits an early onset ataxia with associated features, and the presence of an affected mother and two affected children would make autosomal dominant or mitochondrial inheritance most likely. We have nevertheless considered and sought to exclude those ataxic disorders with associated features, whatever pattern of inheritance has been described.

These disorders include those with known metabolic defects including disorders of pyruvate and lactate metabolism, (pyruvate dehydrogenase, carboxylase deficiencies), hyperammonaemia (ornithine transcarbamoyl synthetase deficiency), the aminoacidurias (Hartnup and isovaleric), abetalipoproteinaemia (Bassen-Kornzweig disease), and vitamin $\mathrm{E}$ deficiency, as well as those with unknown aetiology. These latter disorders include Behr syndrome, an autosomal recessive condition which has associated spasticity and mental retardation, unlike our family. Van Bogaert and Martin ${ }^{2}$ described four families which were thought to follow autosomal recessive inheritance and, in addition to ataxia, deafness, and blindness, they had mental retardation and distal atrophy. Two of the four brothers described by Matthews ${ }^{3}$ were deaf from birth with the onset of ataxia in teenage. Berman et $a l^{4}$ described a sibship who had an early onset ataxia, intellectual deterioration, and neural hearing loss in the first decade, followed by amyotrophy and the development of upper motor neurone signs. Arts et $a l^{5}$ described a disorder with $\mathrm{X}$ linked inheritance which was usually fatal in affected males and associated with absence of myelin in the posterior columns. A number of other $\mathrm{X}$ linked ataxic syndromes have been described and have been reviewed by Arts et $a l^{5}$ but there are clinical differences from our family and, as stated above, the presence of two affected females makes X linked inheritance unlikely.

A number of other associations of early onset cerebellar ataxia have been described in isolated families including the syndrome of cerebellar ataxia with hypogonadism, either alone or with deafness or mental retardation, ${ }^{6}$ the KlippelDurante and Flynn-Aird syndromes with ataxia and sensory hearing loss, the May-White association syndrome comprising ataxia, sensory hearing loss, and myoclonic epilepsy, and finally the mitochondrial cytopathies which typically present with multisystem involvement. While the disorder in our family could be accounted for by a disorder in mitochondrial function, none of the common mitochondrial DNA mutations was identified. Autosomal dominant disorders which were considered include that described by Sylvester ${ }^{7}$ and familial periodic ataxia (FPA). Sylvester ${ }^{7}$ described a family in 1958 with an apparent variant of Friedreich's ataxia which bears some similarity to our family, notably the possible mode of 
inheritance and the progressive optic atrophy and deafness seen in the father, although his six affected children (three sons and three daughters) showed other features including muscle weakness and atrophy and mental retardation. Familial periodic ataxia (FPA), first described by Parker ${ }^{8}$ in 1946, is considered to be a benign paroxysmal disorder characterised by frequent attacks of cerebellar ataxia, dysarthria, and nystagmus which occur intermittently lasting from minutes to hours, and show a good response to acetazolamide. ${ }^{9}$ A subgroup of this heterogeneous disorder has recently been mapped to chromosome $19 \mathrm{p} .{ }^{10}$ In our family, although relapses did occur, the duration of symptoms during the attack and the time interval between attacks is clearly different from that described in FPA; in addition neither optic atrophy nor hearing loss have been described in association with FPA.

A final group of disorders which must be considered are the hereditary motor and sensory neuropathies (HMSN), particularly as a number are associated with additional neurological features. In HMSN type VI, slowly progressive peripheral neuropathy occurs with optic atrophy, and this condition has also been described in association with sensorineural deafness, distal sensory changes, and ataxia. ${ }^{11}$ Both of these conditions are likely to be recessively inherited, and unlike our patients are also characterised by progressive muscular weakness and atrophy. Hagemoser et $a l^{12}$ in 1989 described the triad of motor and sensory neuropathy, optic atrophy, and sensory hearing loss in eight families showing genetic heterogeneity, and identified an autosomal dominant form in patients exhibiting optic atrophy as their first symptom. In HMSN IV (infantile Refsum's disease) cerebellar ataxia occurs in conjunction with peripheral neuropathy, sensorineural deafness, and pigmentary retinopathy ${ }^{13}$; this was again excluded in our patients by the normal serum levels of phytanic acid. Finally, none of our family had a peripheral neuropathy, despite their clinical findings of areflexia and pes cavus. EMG in case 2 was abnormal showing evidence of reinnervation (but no denervation) but the clinical significance of this is unclear. Theoretically, biopsy of a peripheral (for example, the sural) nerve may have provided some more definitive evidence of a peripheral neuropathy; unfortunately the mother (case 2) declined this investigation on herself. Biopsy of the sural nerve in case 1 will be undertaken should this patient have a further relapse.

All three patients described in this case report showed similar features of early normal development and presentation, with cerebellar ataxia secondary to a febrile illness in infancy. In addition, they have generalised hypotonia, areflexia, flexor plantar responses, pes cavus, and all have shown, or are developing, pro- gressive optic atrophy and sensorineural hearing loss. Case 1 is the most severely affected and after three relapses shows marked residual neurological signs. Case 2, at 31 years of age, has only had one major attack and shows similar though milder neurological signs. Case 3, the youngest in the family, has had a single episode and is currently the least severely affected. There is no history of consanguinity, no other family members are known to be affected (either historically or on examination), and the mode of inheritance is likely to be autosomal dominant or mitochondrial through maternal DNA. Extensive investigations have failed to identify an obvious aetiology although mild non-specific EMG abnormalities have been found in the mother. We believe that this family may represent a new syndrome of early onset ataxia with associated features of progressive visual failure, optic atrophy, and deafness. It is currently unclear as to whether this could represent a mitochondrial cytopathy, a variant of FPA, or a new type of HMSN. The progression and severity of the disease shows variability within the family, which is often seen in autosomal dominant and mitochondrial disorders; this variability may alternatively reflect a more severe course in affected males which may itself be directly related to the number and severity of the relapses.

Clearly the recognition of a new syndrome is based solely (at least initially) on clinical criteria and should therefore be regarded as provisional until either further, similar cases are reported, or a specific genetic marker is identified.

The authors are grateful to the late Professor A Harding, Professor D Turnbull, and Dr G Brown for their assistance in the mitochondrial DNA mutation analysis and assays of the the mitochondrial DNA mutation analysis and assays of the
muscle respiratory chain complexes and pyruvate demuscle respiratory chain
hydrogenase in this patient.

1 Harding AE. The hereditary ataxias and related disorders. Clinical neurology and neurosurgery monographs. Vol 6 . Edinburgh: Churchill Livingstone, 1984: 1-11.

2 Van Bogaert L, Martin L. Optic and cochleovestibular degenerations in the hereditary ataxias. Clinico-pathological and genetic aspects. Brain 1974;97:15-40.

3 Matthews WB. Familial ataxia, deaf mutism and muscular wasting. $\mathcal{F}$ Neurol Neurosurg Psychiatry 1950;13:307-11.

4 Berman W, Haslam RHA, Konigsmark BW, Capute AJ Migeon CJ. A new familial syndrome with ataxia, hearing Migeon CJ. A new familial syndrome with ataxia, hearing
loss and mental retardation. Arch Neurl 1973;29:258-61.

5 Arts WFM, Loonen RCA, Sengers A, Slooff JL. X-linked ataxia, weakness, deafness, and loss of vision in early 6 Matthews WB, Rundle AT. Familial cerebellar ataxia and hypogonadism. Brain 1964;87:463-8.

7 Sylvester PE. Some unusual findings in a family with Friedreich's ataxia. Arch Dis Child 1958;33:217-21.

8 Parker HL. Periodic ataxia. Collected Papers Mayo Clinic 1946;38:642-5.

9 Bouchard JP, Roberge C, Van Gelder NM, Barbeau A. Familial periodic ataxia responsive to acetazolamide $\mathrm{Can}$ f Neurol Sci 1984;11:550-3.

10 Teh BT, Silburn P, Lindblad K, et al. Familial periodic cerebellar ataxia without myokymia maps to a 19-cM

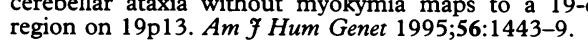

11 Iwashita $\mathrm{H}$, Inoue N, Araki S, Kuroiwa Y. Optic atrophy, neural deafness and distal neurogenic amyotrophy. Report of a family with two affected siblings. Arch Neurol 1970; 22:357-64.

12 Hagemoser K, Weinstein J, Bresnick G, Nellis R, Kirkpatrick S, Pauli RM. Optic atrophy, hearing loss, and periphera neuropathy. Am f Med Genet 1989;33:61-5.

13 Quinlan CD, Martin EA. Refsum's syndrome: report of three cases. F Neurol Neurosurg Psychiatry 1970;33:817-23. 